\title{
Systematic Experimental Investigation of Segregation Direction and Layer Inversion in Binary Liquid-Fluidized Bed
}

\author{
Alberto Di Renzo *(1), Giacomo Rito and Francesco P. Di Maio \\ Dipartimento di Ingegneria Informatica, Modellistica, Elettronica e Sistemistica, Università della Calabria, Via P. \\ Bucci, Cubo 45a, 87036 Rende (CS), Italy; giacomo.r.87@hotmail.it (G.R.); francesco.dimaio@unical.it (F.P.D.M.) \\ * Correspondence: alberto.direnzo@unical.it; Tel.: +39-0984-496654
}

Received: 15 January 2020; Accepted: 30 January 2020; Published: 4 February 2020

\begin{abstract}
Multi-component liquid-fluidized beds are encountered in a variety of industrial processes. Often, segregation severely affects the performance of the process unit. Unfortunately, size-driven and density-driven separation processes may occur with a complex interplay, showing prevailing mechanisms that change with the operating conditions. For example, when the solids exhibit contrasting differences in size and density, even the direction of segregation can turn out hard to predict, giving rise for some systems to the so-called "layer inversion phenomenon". A systematic experimental investigation is presented on 14 different binary beds composed of glass beads and ABS spheres with different size and density ratios and different bed composition. The analysis allows assessing the reliability of a model for predicting the segregation direction of fluidized binary beds (the Particle Segregation Model, PSM). By measurements of the solids' concentration at the surface, expansion/segregation properties and the inversion voidage are compared with the PSM predictions, offering a direct means of model validation. Both the segregation direction throughout the expansion range and the value of the inversion voidage are compared. Extensive qualitative agreement is obtained for 12 out of 14 fluidized mixtures. Quantitatively, the average discrepancy between predicted and measured inversion voidage is below $5 \%$, with a maximum of $17 \%$.
\end{abstract}

Keywords: liquid-fluidization; multi-component beds; mixing; segregation; expansion; layer inversion

\section{Introduction}

Thanks to their homogeneous expansion characteristics and good heat/mass transfer rates, liquid-fluidized beds of one or more particulate materials are utilized in a variety of industrial processes and technologies [1,2]. These include mineral processing [3], sorting/classification [4], coal beneficiation, separation in plastic waste recycling [5], in biochemical reactors $[6,7]$ and in novel biomedical devices [8,9]. Rather often, segregation negatively affects the performance of the process unit, like in biological treatments and bioreactors. In other cases, e.g., in mineral processing and classifiers, solids stratification is the process objective. Unfortunately, size-driven, density-driven, and even shape-driven separation may act at the same time with a complex interplay, showing prevailing mechanisms that change with the operating conditions. For example, when the solids species exhibit contrasting differences in size and density (like in many applications cited above), the mixtures can give rise to the so-called "layer inversion phenomenon", in which a different floating component can be found depending on the fluidization velocity or the mixture composition [10-14] (see Figure 1). Therefore, in such systems, the simple direction of segregation, i.e., which one component tends to separate towards the surface and which one tends to sink to the bottom, can turn out hard to predict. Furthermore, the fluid temperature plays a decisive role, mainly owing to its influence on the fluid 
viscosity [15]. Given the fundamental interest in understanding and characterizing the segregation tendency and the relevance to applications, analytical models of the behavior of a given mixture are very useful.

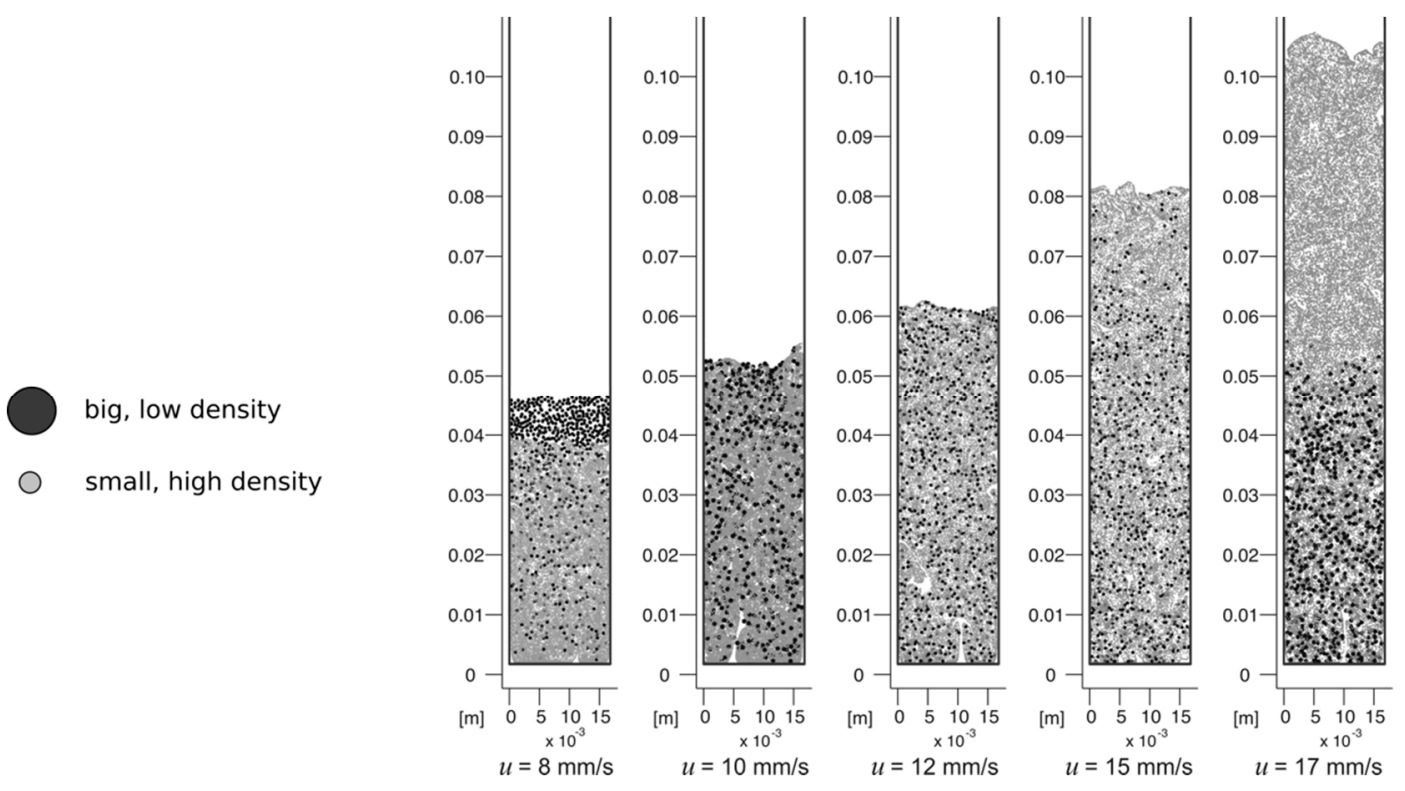

Figure 1. Simulations of the segregation and layer inversion in liquid-fluidized bed. Reprinted from Chemical Engineering Science, 66, Di Renzo A., Cello F., Di Maio F.P., Simulation of the layer inversion phenomenon in binary liquid-fluidized beds by DEM-CFD with a drag law for polydisperse systems, 2945-2958, Copyright (2011), with permission from Elsevier [13].

In the literature, various analytical models have been proposed, most often aimed at calculating the combination of fluid superficial velocity and bed expansion degree, as represented by the overall voidage, leading to the intermediate condition between the two segregation tendencies, named the inversion point (see Figure 1). Apart from discriminating the two segregation directions, this critical point possesses interesting features, for the bed is fully mixed throughout the whole column. Among the most widely used formulations are the "serial model" by Epstein and Pruden [16], the property averaging method introduced by Gibilaro et al. [17] and later extended by Asif [18], and the force balance approach applied separately to the two species by Funamizu and Takakuwa [19]. Most of them require the numerical solution of set of non-linear equations to find the two unknowns (fluid velocity and voidage).

The approach recently introduced by Di Maio and Di Renzo (Particle Segregation Model, PSM) [20] was shown theoretically capable of predicting both the segregation direction and the layer inversion voidage, requiring solely information on the solids properties and bed composition. Based on the hypothesis of bed uniformity and the knowledge of a model for the drag force in size-polidisperse systems, it has been proved possible to directly and analytically predict the segregation tendency of different types of particles in the liquid-fluidized bed.

In the present work, after a short description of the foundations of the PSM model for the inversion voidage in binary liquid-fluidized beds, a systematic experimental investigation on a set of binary mixtures specifically assorted to emphasize the role of the solids' sizes and densities is presented. The results of the inversion voidage are compared to the PSM calculations in an attempt to assess its predicting capabilities.

\section{Direct Prediction of the Inversion Voidage: the Particle Segregation Model (PSM)}

The Particle Segregation Model approach follows from the force equilibrium on one particle immersed in a mixture [20] and is shortly summarized here. The point of departure considers a 
fluidized binary mixture composed of a smaller and denser component and a bigger and less dense component, a necessary but not sufficient condition to exhibit the layer inversion phenomenon. Without loss of generality, the small component is denoted as Species 1 and the bigger one as 2 .

To establish a force balance on one particle species, the foundation of the Particle Segregation Model, expressions for the forces acting on a particle in a mixture of two or more solid components are required, as well as the average properties of the mixture. In the present derivation, the latter are defined as follows:

$$
\begin{aligned}
& \bar{D}=\left(\frac{x_{1}}{D_{1}}+\frac{1-x_{1}}{D_{2}}\right)^{-1} \\
& \bar{\rho}=\rho_{1} x_{1}+\rho_{2}\left(1-x_{1}\right)
\end{aligned}
$$

where $D$ and $\rho$ denote the particle diameter and density, respectively, and $x_{1}$ is the solid volume fraction of solid Species 1 .

To establish the expressions for the force contributions, the following considerations apply. The bed is assumed initially fully mixed and in the fluidized state. Consequently, the pressure gradient equals the apparent bed weight per unit volume. So, the generalized buoyancy force $F_{b}$ acting on a particle of Species 2 can be calculated by

$$
F_{b 2}=V_{2} \nabla p=\frac{\pi}{6} D_{2}^{3}\left(\bar{\rho}-\rho_{f}\right)(1-\varepsilon) g
$$

where $V$ is the particle volume, $\rho_{f}$ is the fluid density and $\varepsilon$ is the voidage, i.e., the volume fraction occupied by the fluid.

According to the findings of the last decade or so, formulations of the drag force $F_{d}$ on an individual particle species in a mixture are available, see e.g., [21-24]. Among these, the simplest one, as introduced in the PSM model by Di Maio and Di Renzo [20], is utilized, which takes the form of the product of a species-dependent coefficient and the average value of the force, $\overline{F_{d}}$. For the particle of Species 2, it reads

$$
F_{d 2}=y_{2}^{2} \overline{F_{d}}
$$

In Equation (4) the species coefficient corresponds to the polydispersion index $y_{i}$, defined as

$$
y_{i}=\frac{D_{i}}{\bar{D}}
$$

The average drag force in the system, $\overline{F_{d}}$, can be related to the sum of all drag forces exerted on the individual particle species by

$$
\overline{F_{d}}=\sum_{i} \frac{x_{i}}{y_{i}^{3}} F_{d i}
$$

However, since the mixture is assumed fully fluidized, the average force can be more conveniently expressed as the value balancing the apparent weight of an average particle, i.e.,

$$
\overline{F_{d}}=\varepsilon\left(\bar{\rho}-\rho_{f}\right) \frac{\pi}{6} \bar{D}^{3} g
$$

Therefore, the force balance on Species 2 reads

$$
V_{2} \nabla p+F_{d 2}=\rho_{2} \frac{\pi}{6} D_{2}^{3} g
$$

which, upon substitution of the generalized buoyancy force, Equation (3), and the drag force, Equations (4) and (7), can be rearranged to lead to the following prediction formula for the equilibrium voidage:

$$
\varepsilon_{i n v}=\frac{1-\bar{s}}{1-\bar{d}}
$$


In Equation (9) the following definitions of the average size ratio and net density ratio appear:

$$
\bar{d}=\frac{\bar{D}}{D_{2}}
$$

and

$$
\bar{s}=\frac{\rho_{2}-\rho_{f}}{\bar{\rho}-\rho_{f}}
$$

An equivalent expression of the inversion voidage in terms of species size and density ratios is:

$$
\varepsilon_{i n v}=\frac{x_{1}-\left(1-x_{1}\right) d}{x_{1}-\left(1-x_{1}\right) s}
$$

where

$$
d=\frac{D_{1}}{D_{2}}
$$

and

$$
s=\frac{\rho_{2}-\rho_{f}}{\rho_{1}-\rho_{f}}
$$

It shall be noted that the voidage value obtained by the Particle Segregation Model, Equation (9) or Equation (12), named the force balance on Species 2 under the assumption of a fluidized and fully mixed binary mixture, can be interpreted as the inversion condition voidage, hence the subscript inv. Indeed, a peculiar condition occurs for which the Species 2 particles are both under equilibrium as a species and within a system that is globally at equilibrium (suspended). As a consequence, there is no drive to internal segregation during fluidization. Note that owing to the total bed equilibrium, also Species 1 particles have to be under force equilibrium, so the full system remains mixed. On the contrary, at voidage values different than $\varepsilon_{\text {inv }}$ some segregation occurs, in the "direction" that depends on the sign of the prevailing force. Within the suspended bed, one species will be found with hydrodynamic forces dominating (the one segregating to the top, or flotsam) and the other one with gravity force prevailing (the one settling to the bottom, or jetsam). A map in terms of the average size and density ratios like the one in Figure 2 can be drawn.

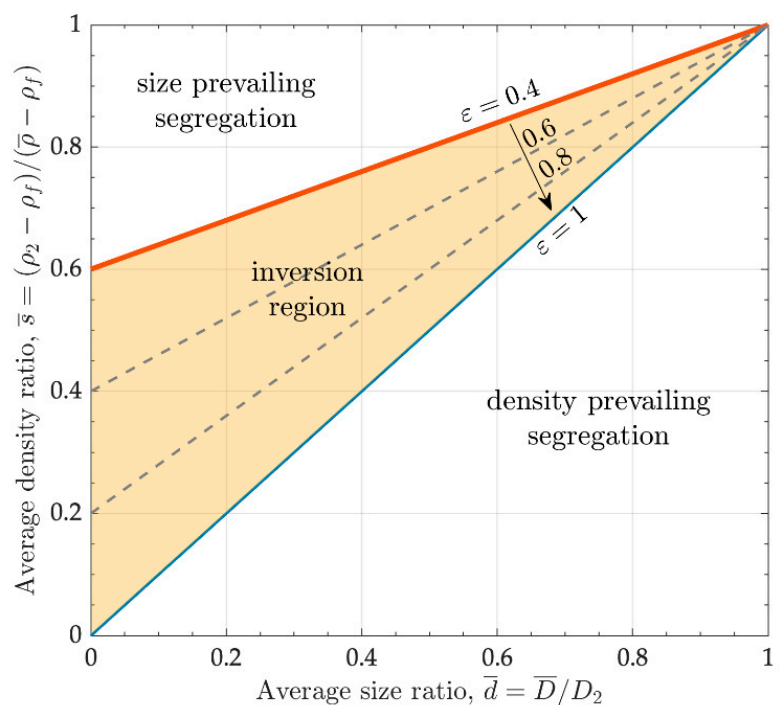

Figure 2. Layer inversion zone in the average density vs. average diameter ratio map. Three distinct regions appear: the one where the denser component tends to settle and the lighter one tends to float (density prevailing); the one where the finer component tends to float and the bigger one to settle (size prevailing); the intermediate region where the segregation direction can change with expansion (layer inversion). 
Differently from other macroscopic models, in which the inversion conditions of voidage and velocity are strongly coupled, Equation (9) leads to the direct prediction of the inversion voidage, lending itself to a relatively easy experimental verification. In addition, there are no fitting or otherwise adjustable parameters.

\section{Experimental Setup, Methods and Materials}

Different binary mixtures potentially exhibiting layer inversion have been tested in a water fluidization rig. The experimental facility is composed of a transparent PMMA, $10 \mathrm{~cm}$ diameter, $1.75 \mathrm{~m}$ height fluidization column, inserted in a water recirculation loop, as illustrated in Figure 3. The distribution section is composed by a plenum chamber filled with a static bed of $10 \mathrm{~mm}$ glass beads followed by a perforated steel plate with $400 \mu \mathrm{m}$ diameter holes on a $1.5 \mathrm{~mm}$ pitch square mesh. Recirculated water at temperature within the range $20-22{ }^{\circ} \mathrm{C}$ was used as fluidizing medium. Flowrate is ensured by two pumps, Salmson NSB-S 40-25 and Calpeda MXH 804, depending on the required flow range, and measured by a battery of four rotameters (model FTV215, FTV225, FTV240 and FDZ1, Officine Orobiche Srl). The measurable water flow ranges from 0.016 to $8 \mathrm{~m}^{3} / \mathrm{h}$. Bed height is acquired by direct observation of the liquid level and two meter-tapes attached to the column sides.

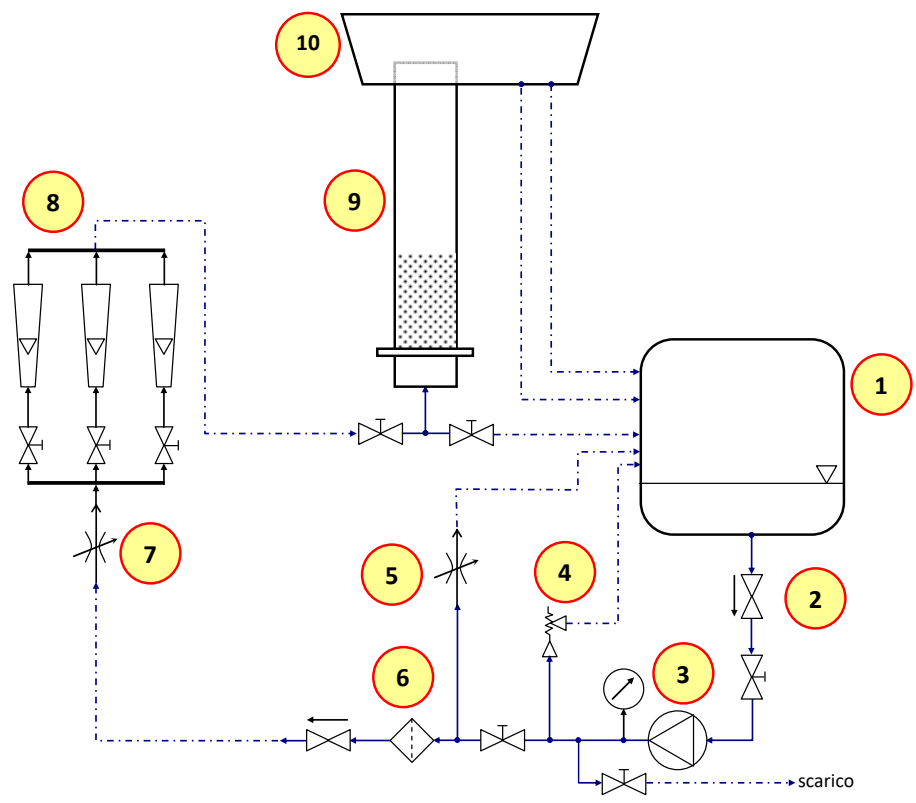

Figure 3. Experimental water-fluidization rig. 1. Tank; 2. Check valve; 3. Pump; 4. Overpressure valve; 5. By-pass valve; 6. Filter; 7 . Fine regulation valve; 8 . Rotameters; 9 . Fluidization column (10 cm ID, $1.75 \mathrm{~m}$ height); 10 . Collection tank.

The overall bed voidage is computed based on the bed height and the known solids mass and density. Solids volume fraction at the surface is evaluated by withdrawing a sample of solids from the fluidized bed surface, using a cup shaped device (volume $=62.8 \mathrm{~mL}$ ), sieving and weighing the solids. Typical solid mass sampled ranges $10-40 \mathrm{~g}$, depending on the expansion degree. After each such measurement, the solids are reinserted in the bed to maintain the initial solids amount.

Independence of the effect of the distributor is achieved by initial bed heights with aspect ratio $\frac{H_{0}}{D_{c}}>1.8$. Measured packed bed height ranged $18-35 \mathrm{~cm}$ with an average voidage of 0.42 . The total column height sets the following corresponding maximum experimental voidage values: $\varepsilon_{\max }=$ $0.93-0.88$.

The pouring arrangement of the solids was sequential, following the order in the typical evolution of the layer inversion phenomenon (see Figure 1), i.e., the fine glass beads first (at the bottom) and then the larger plastic spheres (at the top). 
To investigate the role of particle size and density in the mixture, spherical particles are used as bed materials, including different size cuts of glass beads (SiLi-Beads) and bigger, calibrated size particles made of a different density, Acrylonitrile Butadiene Styrene (ABS) co-polymers and different amounts of $\mathrm{BaSO}_{4}$ (from reseller of common ammunition for airsoft guns). The density of the sphere was as declared by the manufacturer. The average particle size was calculated by dynamic image particle size analysis (Sympatec QICPIC). The codes and properties of the mixture components are listed in Table 1.

Table 1. Glass beads (GB) and calibrated plastic spheres (PS) properties.

\begin{tabular}{cccc}
\hline Code & Densit $\left.\mathbf{( k g} / \mathbf{m}^{\mathbf{3}}\right)$ & Nominal Size Cut $(\mathbf{m m})$ & Average Size $(\mathbf{m m})$ \\
\hline GB1 & 2500 & $0.60-0.81$ & 0.87 \\
GB2 & 2500 & $1.40-2.0$ & 2.12 \\
GB3 & 2500 & $2.40-2.90$ & 2.80 \\
GB4 & 2500 & $2.85-3.45$ & 3.18 \\
GB5 & 2500 & $3.80-4.40$ & 4.21 \\
PS1 & 1451 & 5.95 & 5.95 \\
PS2 & 1632 & 5.95 & 5.95 \\
PS3 & 1814 & 5.95 & 5.95 \\
PS4 & 2086 & 5.95 & 5.95 \\
PS5 & 2267 & 5.95 & 5.95 \\
\hline
\end{tabular}

\section{Results and Discussion}

\subsection{Expansion Properties of the Mixture Components}

Individual component expansion tests have been carried out. Representative results are shown in Figure 4a,b for one glass bead cut, GB4, and one plastic sphere type, PS4, respectively. The expansion rate and intersection with nominally infinite expansion $(\varepsilon=1)$ are compared against the predictions of the Richardson-Zaki equation with the wall correction factor $k$ [6], i.e.,

$$
u=k u_{t 0} \varepsilon^{n}
$$

in which the parameters are the slope $n$ and the intercept $k u_{t 0}$, where $u_{t 0}$ is the particle terminal velocity. The fitted values of the slope and intercept are presented in Table 2, together with the values predicted using the correlations described in Epstein [6] (Section 4.3). Some scatter in Figure $4 \mathrm{~b}$ can be attributed to a non-uniform bed height at high velocities and with big particles.

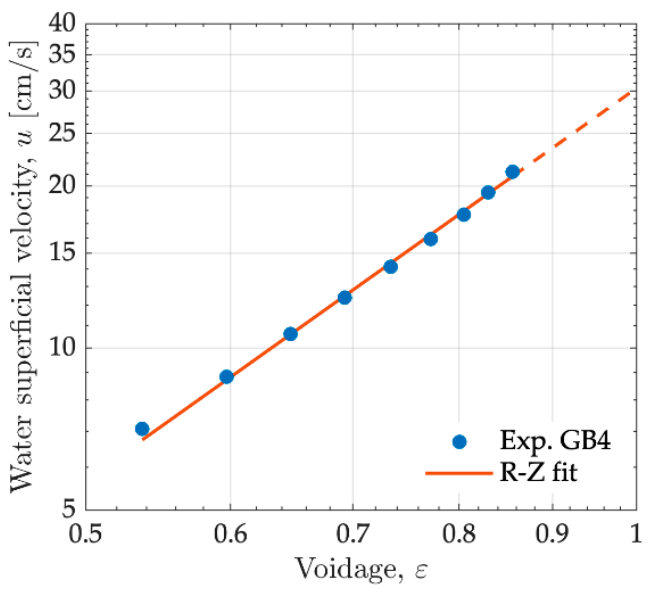

(a)

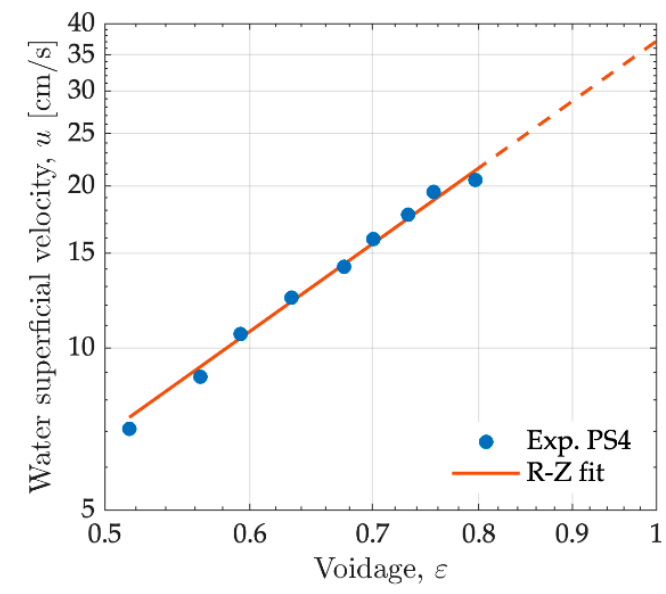

(b)

Figure 4. Expansion properties of one sample of glass beads, GB4, (a) and plastic spheres, PS4, (b). The parameters of the fitting lines are reported in Table 2 and compared with empirical correlations. 
Table 2. Slope and intercept parameters of the fitting lines for the expansion of solids GB4 (Figure 4a) and PS4 (Figure 4b). Correlations for the Richardson-Zaki exponent, terminal velocity and wall correction factor, see Equation (15), are reported in Epstein [6] (Chapter 26).

\begin{tabular}{cccccc}
\hline System & $\begin{array}{c}\text { Fitting Line } \\
\text { Slope (-) }\end{array}$ & $\begin{array}{c}\text { Richardson-Zaki } \\
\text { Exponent (-) }\end{array}$ & $\begin{array}{c}\text { Fitting Line } \\
\text { Intercept }(\mathbf{m m} / \mathbf{s})\end{array}$ & $\begin{array}{c}\text { Terminal } \\
\text { Velocity (mm/s) }\end{array}$ & $\begin{array}{c}\text { Wall Correction } \\
\text { Factor (-) }\end{array}$ \\
\hline GB4 & 2.42 & 2.43 & 303 & 381 & $0.75-0.85$ \\
PS4 & 2.43 & 2.41 & 371 & 444 & $0.71-0.80$ \\
\hline
\end{tabular}

\subsection{Segregation Direction and Layer Inversion}

A typical fully fluidized mixture with the plastic spheres appearing strongly segregated at the top is shown in Figure 5a, a condition of bed mixing in Figure $5 b$ and a completely inverted segregated mixture in Figure 5c.

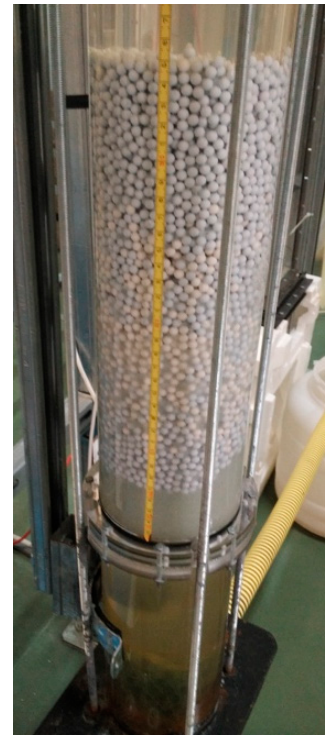

(a)

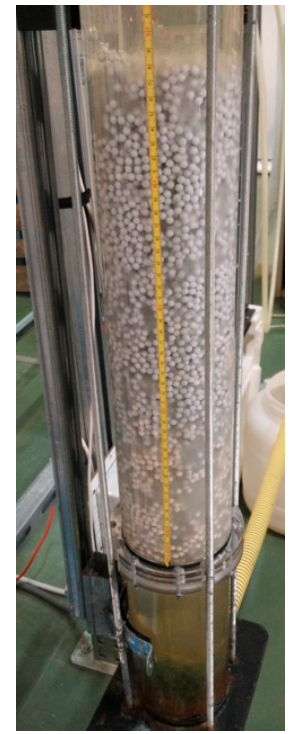

(b)

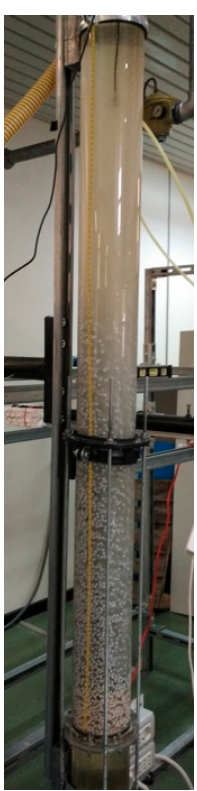

(c)

Figure 5. Photographs of a typical fluidization behavior for a mixture of fine glass beads (GB1) and light plastic spheres (PS1): (a) fully segregated condition at low water velocity; (b) mixed condition at intermediate velocity; (c) expanded inverted layers condition at high velocity.

Several combinations of the materials presented in Section 3 have been subjected to the systematic analysis of the segregation direction and possible occurrence of the layer inversion phenomenon. They are denoted by a string representing the codes of the glass beads $x$, the plastic spheres $y$ and the volume fraction of the former $z . z z$, e.g., "GB $x_{-} \mathrm{PS} y \_X 1 \_z . z z$ ". The list of the combinations examined is reported in Table 3, along with the mixture composition $x_{1}$, absolute size and density ratios $d$ and $s$, species-to-average size and density ratios $\bar{d}$ and $\bar{s}$, and the predicted value of the inversion voidage calculated by Equation (9) or Equation (12). In addition, the inversion voidage experimentally determined (as described below) is reported whenever it was observable in the investigated range of operating variables and the corresponding percent deviation of the model predictions. 
Table 3. Combinations of solids and mixture properties of the investigated systems, with composition, absolute species and species-to-average size and density ratios, inversion voidage predicted by Equation (9) or Equation (12) (Particle Segregation Model, PSM), the experimentally determined inversion voidage and the deviation between the last two.

\begin{tabular}{|c|c|c|c|c|c|c|c|c|}
\hline Code & $\begin{array}{c}\text { GB vol. Fraction } \\
x_{1}\end{array}$ & $\begin{array}{c}\text { Species Size Ratio } \\
d\end{array}$ & $\begin{array}{c}\text { Species Density Ratio } \\
s\end{array}$ & Species to Avg. Size Ratio & Species to Avg. Density Ratio & $\begin{array}{c}\text { Inversion Voidage (PSM) } \\
\varepsilon_{i n v}\end{array}$ & $\begin{array}{c}\text { Inversion Voidage (exp.) } \\
\varepsilon_{i n v}\end{array}$ & $\begin{array}{l}\text { Deviation } \\
\Delta \varepsilon_{\text {inv }}(\%)\end{array}$ \\
\hline GB3_PS5_X1_0.48 & 0.48 & 0.47 & 0.91 & 0.65 & 0.92 & $<0.40$ & $<0.40$ & - \\
\hline GB4_PS5_X1_0.48 & 0.48 & 0.53 & 0.91 & 0.71 & 0.92 & $<0.40$ & $<0.40$ & - \\
\hline GB3_PS4_X1_0.45 & 0.45 & 0.47 & 0.83 & 0.66 & 0.85 & 0.44 & 0.53 & $17 \%$ \\
\hline GB1_PS2_X1_0.30 & 0.30 & 0.15 & 0.65 & 0.36 & 0.71 & 0.46 & $<0.40$ & - \\
\hline GB5_PS5_X1_0.48 & 0.48 & 0.71 & 0.91 & 0.83 & 0.92 & 0.49 & 0.51 & $4 \%$ \\
\hline GB1_PS1_X1_0.08 & 0.08 & 0.15 & 0.58 & 0.68 & 0.84 & 0.49 & 0.51 & $4 \%$ \\
\hline GB4_PS4_X1_0.45 & 0.45 & 0.53 & 0.83 & 0.72 & 0.85 & 0.52 & 0.51 & $-2 \%$ \\
\hline GB1_PS1_X1_0.31 & 0.25 & 0.15 & 0.58 & 0.41 & 0.63 & 0.62 & 0.62 & $0 \%$ \\
\hline GB1_PS1_X1_0.58 & 0.58 & 0.15 & 0.58 & 0.23 & 0.43 & 0.74 & 0.76 & $3 \%$ \\
\hline GB3_PS3_X1_0.42 & 0.42 & 0.47 & 0.73 & 0.68 & 0.74 & 0.82 & 0.80 & $-3 \%$ \\
\hline GB2_PS2_X1_0.30 & 0.30 & 0.36 & 0.65 & 0.65 & 0.71 & 0.83 & $\left(>\varepsilon_{\max }\right)$ & - \\
\hline GB5_PS4_X1_0.45 & 0.45 & 0.71 & 0.83 & 0.84 & 0.85 & 0.93 & $\left(>\varepsilon_{\max }\right)$ & - \\
\hline GB4_PS3_X1_0.42 & 0.42 & 0.53 & 0.73 & 0.73 & 0.74 & 0.98 & $\left(>\varepsilon_{\max }\right)$ & - \\
\hline GB5_PS3_X1_0.42 & 0.42 & 0.71 & 0.73 & 0.85 & 0.74 & $>1.00$ & $\left(>\varepsilon_{\max }\right)$ & - \\
\hline
\end{tabular}


All the investigated mixtures are represented as points on the inversion map (Figure 6), to highlight the prediction of the PSM model. Despite the combination of particles of large size and low density with particles of reverted properties, not all mixtures lay within the inversion zone and, therefore, not all of them are predicted to exhibit layer inversion. To investigate the separate effect of the concentration, three systems show mixtures composed by different amounts of the same pair of solids, GB1 and PS1 with $x_{1}=0.08,0.31$ and 0.58 (see Table 3 and Figure 6).

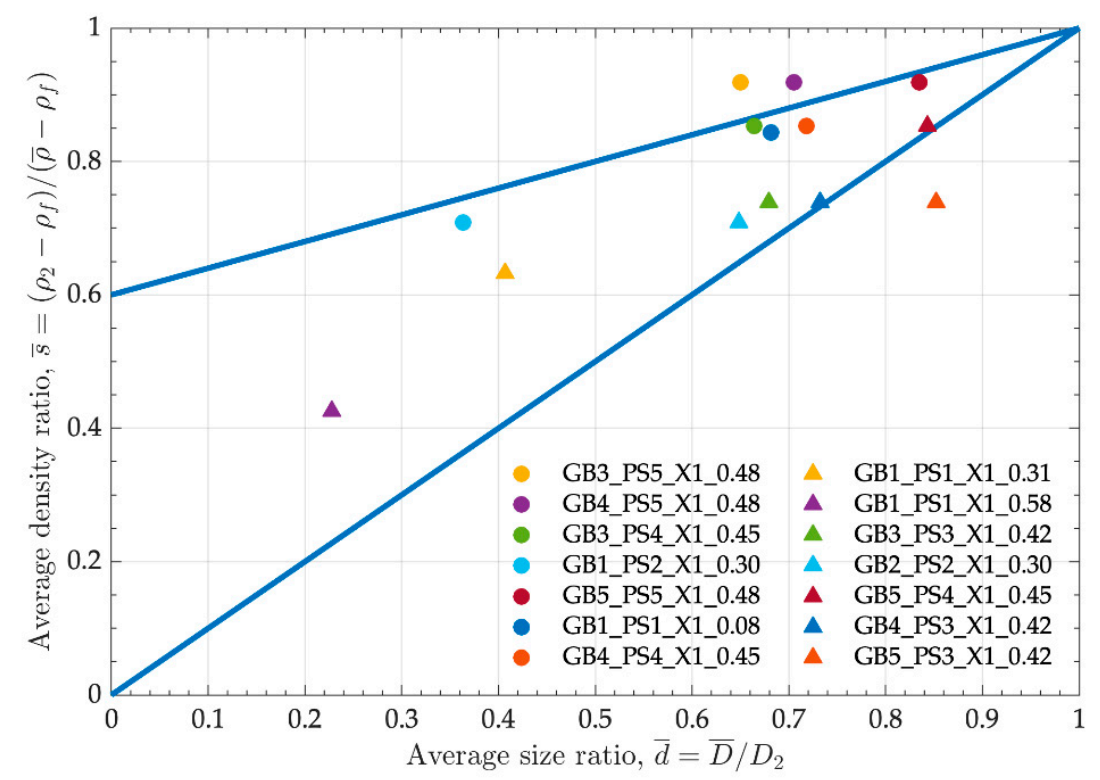

Figure 6. Representative points for the mixtures on the density vs. size ratio map (see Figure 2).

During bed expansion, the solid volume fractions of the uppermost layer were experimentally measured as a function of the water superficial velocity. These values are compared with the nominal volume fraction of the overall mixture. Under the commonly observed hypothesis that segregation manifests with two layers of different concentrations of the two solids, if the top layer concentration profile of glass beads at some degree of expansion equals the nominal mixture value, at that point the fluidized bed can be assumed to be fully mixed. In other words, the fraction of glass beads changes from less than to greater than its nominal (i.e., overall) concentration. That peculiar, intermediate condition is judged as corresponding to the layer inversion point for the system.

Figure 7 shows two examples of a mixture whose profile of the top layer volume fraction with voidage is compared with the nominal mixture concentration (red horizontal arrow). Based on the measurements, it is concluded that the mixture GB4_PS3_X1_0.42 does not exhibit a cross point (Figure 7a), indicating no segregation direction inversion conditions (not in the expansion range technically observable). The mixture GB5_PS5_X1_0.48 does show segregation direction inversion (Figure $7 b$ ), as witnessed by the intersection between the volume fraction profile and the nominal concentration. This change of segregation direction does not necessarily imply complete layer inversion, since nominal layer inversion assumes that a pure layer of one component, typically the larger one, appears at the top $\left(x_{1}=0\right)$ at low velocities and a pure layer of the other one $\left(x_{1}=1\right)$ at higher velocities. In other words, while layer inversion would require the full transition from the first condition sketched in Figure 1 to the last one, the concentration profile shown in Figure $7 \mathrm{~b}$ represents a transition that goes from the second sketch to the fourth condition sketched in Figure 1, no further than that even at high expansion degrees. As shown later (see e.g., Figure 8), most of the systems exhibit the full layer inversion transition. 


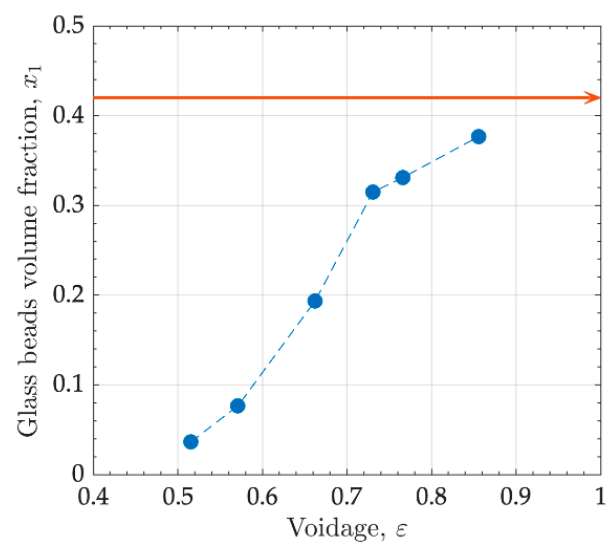

(a)

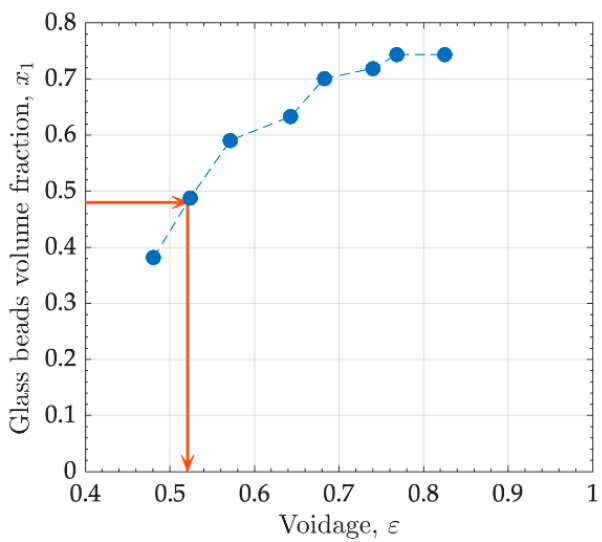

(b)

Figure 7. Representative trends of the concentration of the uppermost layer as a function of the expansion (voidage). As indicated by the presence or absence of the intersection of the profile with the nominal concentration (red arrow), for system GB4_PS3_X1_0.42 (a) the inversion condition is not reached in the investigated range and for system GB5_PS5 _X1_0.48 (b) layer inversion is found to occur.

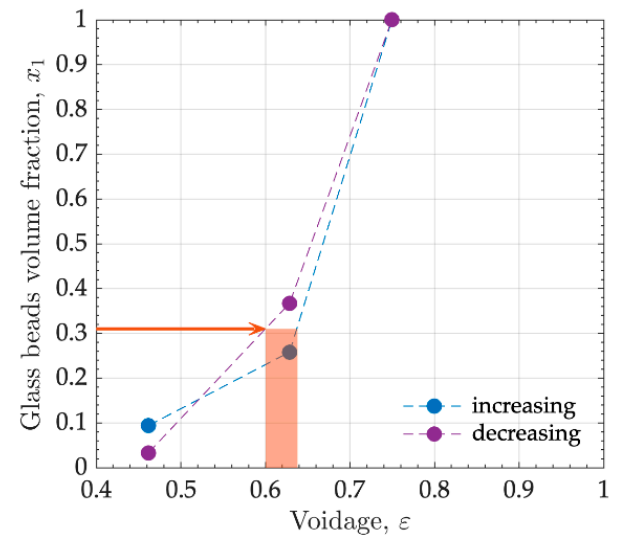

(a)

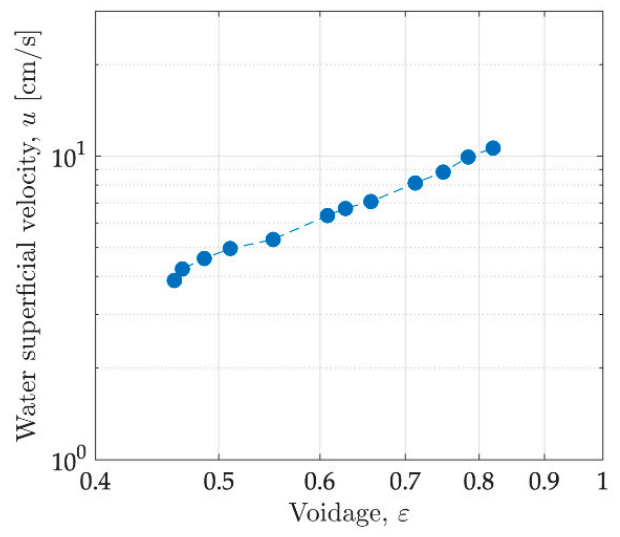

(c)

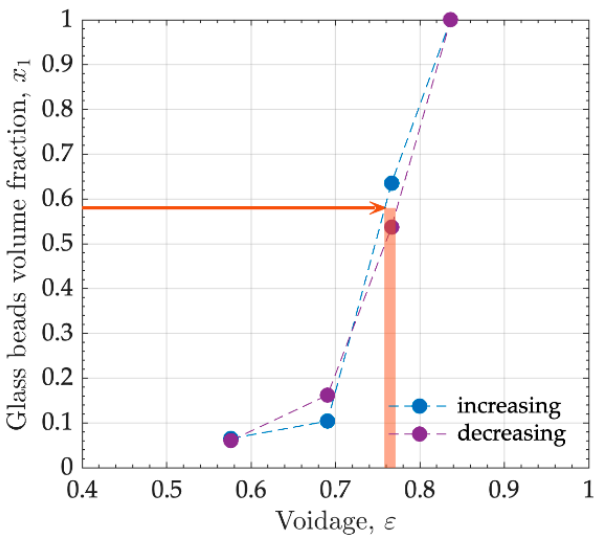

(b)

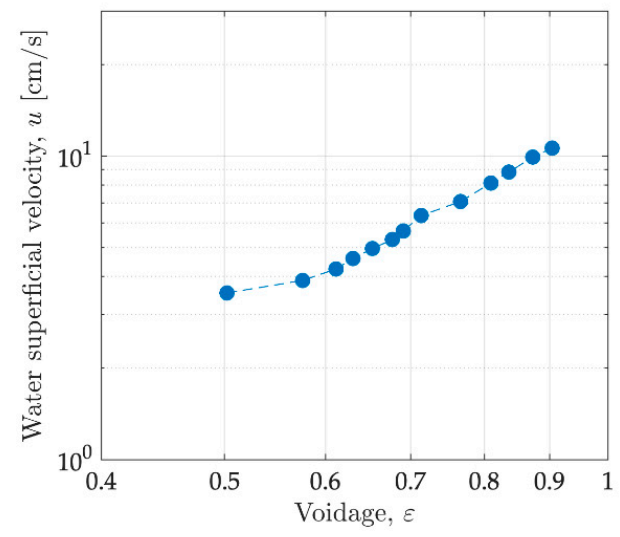

(d)

Figure 8. Determination of the experimental inversion voidage average and uncertainty range and mixture expansion characteristics. Example of the concentration of the uppermost layer as a function of the voidage for the system GB1_PS1_X1_0.31 (a) and GB1_PS1_X1_0.58 (b), corresponding velocity-voidage relationships (c) and (d), respectively. 
In all cases for which segregation direction or full layer inversion occurs, the inversion voidage $\varepsilon_{\text {inv }}$ is determined as the abscissa value of the intersection point. Generally, the average value of multiple runs is taken, for example collecting data at increasing and decreasing velocity (see Figure 8). In Figure 8 , concentration vs. voidage relationship for the mixtures GB1_PS1_X1_0.31 and GB1_PS1_X1_0.58 and their velocity vs. voidage relationships are reported, with the latter plots in logarithmic scale, in analogy to the expansion of mono-component beds. In comparison with Figure 7, the rapid and complete segregation direction reversal exhibited with the expansion degree (voidage) is noticed. This can be attributed to the rather extreme values of the size and density ratios, as shown by the bottom-left position of the corresponding point on the segregation map (Figure 6).

For the systems considered in Figure $8 \mathrm{a}, \mathrm{b}$ the estimated uncertainty on the voidage is less than $2 \%$. Considering all tests, the experimental variability on $\varepsilon_{i n v}$ was limited to $8 \%$.

Examining the expansion characteristics of the two mixtures (Figure $8 \mathrm{c}, \mathrm{d}$ ), it shall be noticed that the voidage is not generally uniform, as the segregation may cause a vertical profile with local differences. Consequently, the reported voidage is the value corresponding to the total amount of void space divided by the total bed volume. At the inversion point, however, the mixture is by definition uniformly mixed. Therefore, the voidage at the inversion point should be considered a uniform property of the mixture.

\subsection{Overall Comparison with PSM Predictions}

The comparison of predicted and measured segregation behavior is reported in Figure 9. In the experimentally investigated range of expansion voidage, the match for the qualitative segregation direction and the occurrence of the layer inversion and is found for 12 out of 14 systems. The two systems for which incorrect predictions are obtained are GB1_PS2_X1_0.30, uniform segregation dominated by size instead of inversion theoretically predicted, and GB2_PS2_X1_0.30, uniform segregation dominated by density instead of inversion theoretically predicted. In both cases, the theoretical inversion voidage is close to the limiting expansion conditions, i.e., close to 0.4 and $\varepsilon_{\max }$, respectively. It is presumable that such a situation can be hard to check experimentally, as for example near the packing limit (i.e., low voidage), the segregation tendency can be hindered or strongly delayed due to the low particle mobility.

The last three systems in Figure 9 all show consistent observations and PSM theoretical predictions, although the experimental measurements only reported (density) segregation. The calculated inversion voidage lies at an expansion degree above $\varepsilon_{\max }$. It is worth mentioning that the observed concentration profile with voidage of these systems shows an increasing trend, in agreement with the predictions. The fact that in the investigated range the observed top layer concentration never reached the nominal mixture concentration does not exclude high probabilities to be captured at higher voidage values. This conclusion is corroborated by examining the concentration profile of these systems. One of these is plotted in Figure 7a, where the concentration profile can be estimated to reach the nominal mixture at an expansion voidage $\varepsilon_{i n v}>0.9$, in reasonable agreement with the PSM predictions.

For all cases when the inversion condition is explicitly observed, the PSM model predictions show an average discrepancy on $\varepsilon_{i n v}$ below $5 \%$, with a maximum of $17 \%$. All predicted and measured values are reported in Table 3.

Interestingly, for the three mixtures involving PS1 and GB1 in Figure 9, which differ only in the concentration of the two components $\left(x_{1}=0.08,0.31\right.$ and 0.58 , respectively), the experimental and predicted inversion voidage values exhibit remarkably similar behavior, both qualitative and quantitative.

Aside from the inherent significance of the systematic analysis presented, the results discussed earlier provide a substantial corroboration of the quality of the predictions achievable using the PSM model. It is worth recalling that the ability to estimate the voidage at the inversion conditions independently of the corresponding velocity is a unique feature over previous models. For example, in the results presented by Escudiè and Epstein [25] specifically on the inversion voidage, it was calculated knowing the inversion velocity. 


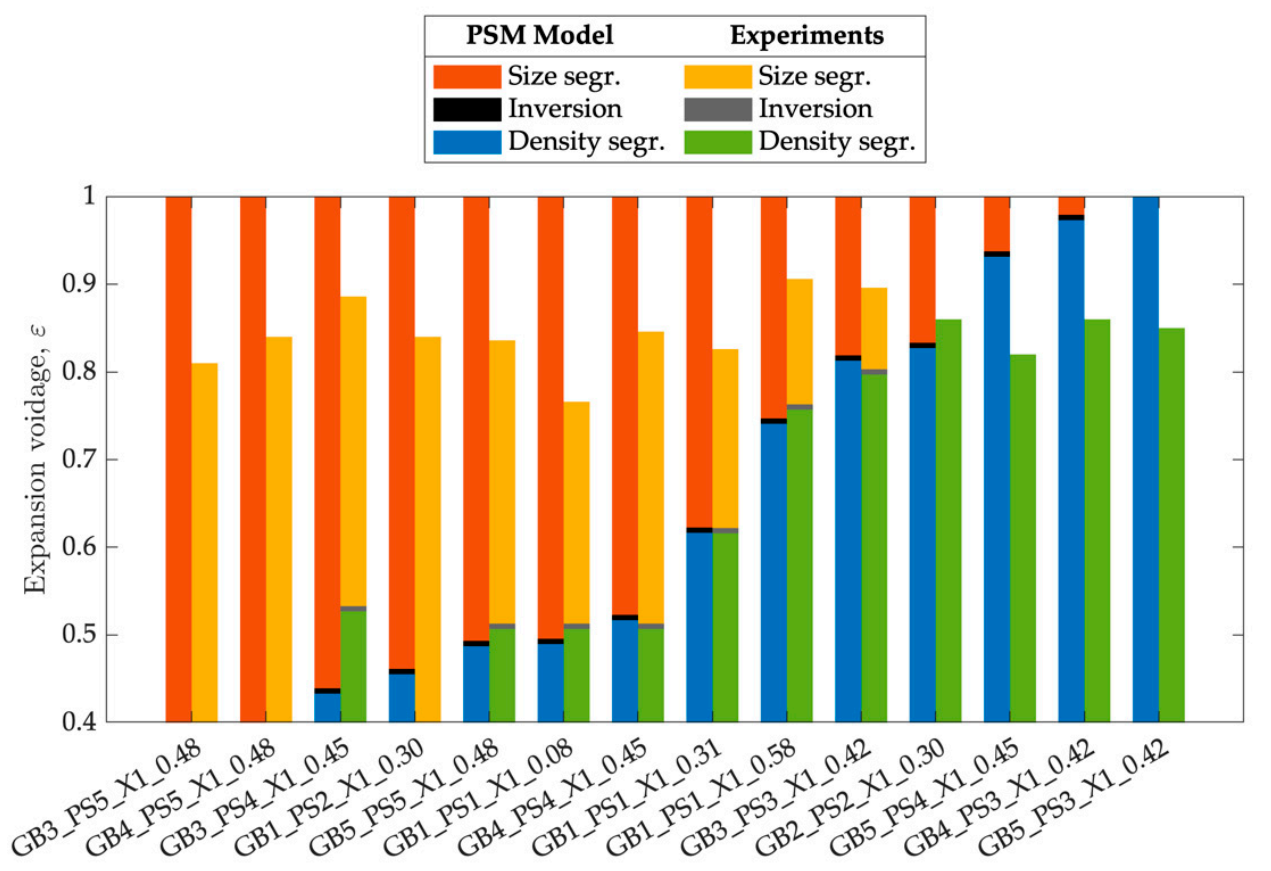

Figure 9. Histogram plot comparing the segregation direction and the inversion voidage between the PSM model predictions and the experimental measurements. Color codes are illustrated in the legend: blue and green indicate segregation dominated by density, red and orange segregation dominated by size and the black and grey lines denote the inversion voidage. Note that the maximum height of the experimental bar indicates the highest voidage achieved in the column for each test. The systems are sorted by increasing predicted inversion voidage. Solids and mixture properties are available in Tables 1 and 3.

Overall, the comparison of the systematic investigation with the PSM predictions shows that the inversion map shown in Figure 2 provides a simple and relatively effective way to predict the segregation direction of a given mixture (in terms of solids' size and density ratios and composition) and, in case of possible inversion, to estimate its voidage.

\section{Conclusions}

The segregation behavior of binary mixtures composed of small denser particles and bigger, less dense particles in water-fluidized beds was systematically investigated by measuring the tendency exhibited over a relatively wide range of expansion degree. In particular, the occurrence of the layer inversion phenomenon and its characteristic voidage value were experimentally determined for different particle size, density and mixture concentration.

The selection of systems was driven by taking into consideration the predictions of the Particle Segregation Model. In theory, the model is able to capture the influence of solids' properties and mixture concentration, allowing parameter-free analytical prediction of the inversion voidage, irrespective of the corresponding velocity.

Experimental determination of the surface solid concentration allowed volume fraction vs. average voidage plots to be obtained for 14 different mixtures involving 5 cuts of glass beads and 5 calibrated plastic spheres with different densities. Despite the simple procedure to determine the concentration, repeatability tests showed reproducibility of the voidage to within $8 \%$. PSM ability to capture the occurrence of layer inversion phenomena appeared reliable both for exhibiting and non-exhibiting systems (12 out of 14). Quantitative agreement, with an average below 5\% and a maximum of $17 \%$, appeared encouraging, particularly considering the simplicity and the lack of adjustable parameters of the PSM model formulation. 
Author Contributions: Conceptualization, A.D.R. and F.P.D.M.; data curation, G.R.; formal analysis, A.D.R. and F.P.D.M.; investigation, G.R.; methodology, A.D.R. and F.P.D.M.; writing-original draft, A.D.R.; writing-review \& editing, F.P.D.M. All authors have read and agreed to the published version of the manuscript.

Funding: This research was funded by University of Calabria.

Acknowledgments: The support of the PON SILA project (code: PONa3_00341 funded by Italian ministries MIUR and MISE) with the availability of the Sympatec QICPIC measurement device is gratefully acknowledged.

Conflicts of Interest: The authors declare no conflict of interest.

\section{Abbreviations}

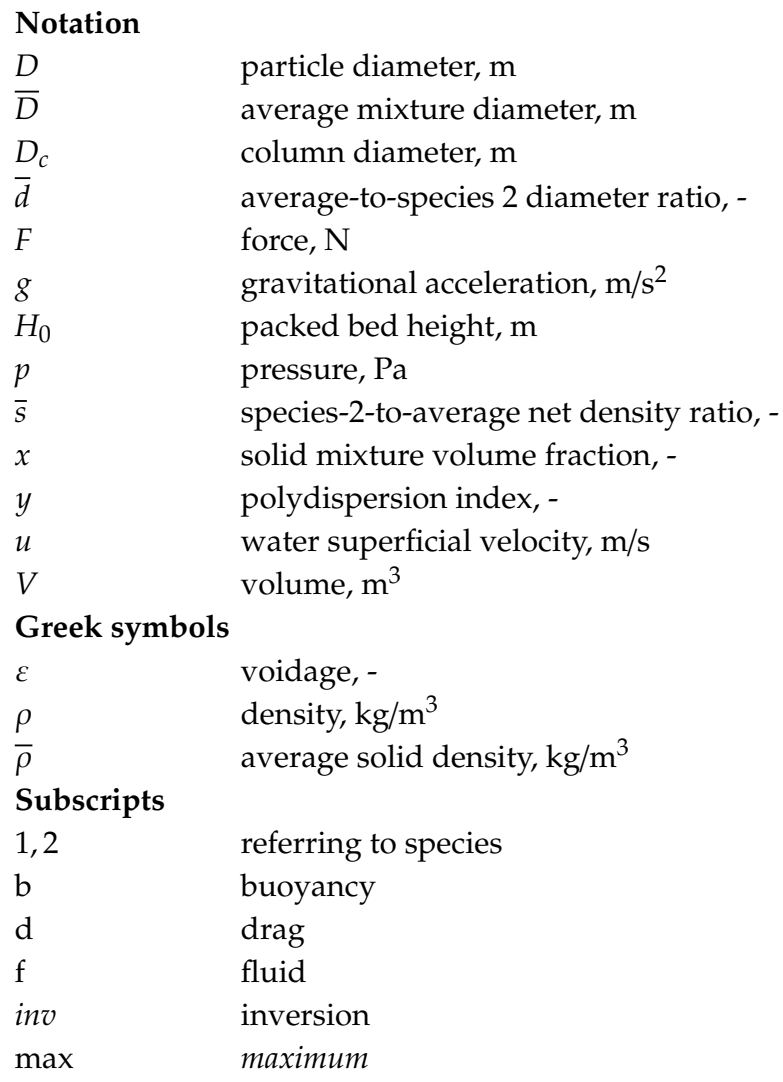

\section{References}

1. Di Felice, R. Hydrodynamics of Liquid Fluidisation. Chem. Eng. Sci. 1995, 50, 1213-1245. [CrossRef]

2. Epstein, N. Applications of Liquid-Solid Fluidization. Int. J. Chem. React. Eng. 2003, 1, 1-16. [CrossRef]

3. Sahu, A.K.; Tripathy, A.; Biswal, S.K. Study on particle dynamics in different cross sectional shapes of air dense medium fluidized bed separator. Fuel 2013, 111, 472-477. [CrossRef]

4. Tanaka, Z.; Song, X. Continuous separation of particles by fluidized beds. Adv. Powder Technol. 1996, 7, $29-40$. [CrossRef]

5. Tatemoto, Y.; Higashino, T.; Suzuki, Y.; Michikoshi, T.; Maeda, S.; Bando, Y. Prediction of the Behavior of a Liquid-Fluidized Bed of Inert Particles Used for Separation by Density. Chem. Eng. Technol. 2011, 34, 877-885. [CrossRef]

6. Epstein, N. Liquid-solids Fluidization. In Handbook of Fluidization and Fluid-Particle Systems; Yang, W.-C., Ed.; Marcel Dekker Inc.: New York, NY, USA, 2003.

7. Di Felice, R.; Nicolella, C.; Rovatti, M. Mixing and segregation in water fluidised-bed bioreactors. Water Res. 1997, 31, 2392-2396. [CrossRef]

8. Coward, S.M.; Legallais, C.; David, B.; Thomas, M.; Foo, Y.; Mavri-Damelin, D.; Hodgson, H.J.; Selden, C. Alginate-encapsulated HepG2 Cells in a Fluidized Bed Bioreactor Maintain Function in Human Liver Failure Plasma. Artif. Organs 2009, 33, 1117-1126. [CrossRef] 
9. Naghib, S.D.; Pandolfi, V.; Pereira, U.; Girimonte, R.; Curcio, E.; Di Maio, F.P.; Legallais, C.; Di Renzo, A. Expansion properties of alginate beads as cell carrier in the fluidized bed bioartificial liver. Powder Technol. 2017, 316, 711-717. [CrossRef]

10. Moritomi, H.; Iwase, T.; Chiba, T. A comprehensive interpretation of solid layer inversion in liquid fluidised beds. Chem. Eng. Sci. 1982, 37, 1751-1757. [CrossRef]

11. Vivacqua, V.; Vashisth, S.; Hébrard, G.; Grace, J.R.; Epstein, N. Characterization of fluidized bed layer inversion in a 191-mm-diameter column using both experimental and CPFD approaches. Chem. Eng. Sci. 2012, 80, 419-428. [CrossRef]

12. Di Felice, R.; Gibilaro, L.G.; Foscolo, P.U. On the inversion of binary-solid liquid fluidised beds. Chem. Eng. Sci. 1988, 43, 979-981. [CrossRef]

13. Di Renzo, A.; Cello, F.; Di Maio, F.P. Simulation of the layer inversion phenomenon in binary liquid-fluidized beds by DEM-CFD with a drag law for polydisperse systems. Chem. Eng. Sci. 2011, 66, 2945-2958. [CrossRef]

14. Abbaszadeh Molaei, E.; Yu, A.B.; Zhou, Z.Y. Investigation of causes of layer inversion and prediction of inversion velocity in liquid fluidizations of binary particle mixtures. Powder Technol. 2019, 342, 418-432. [CrossRef]

15. Escudié, R.; Epstein, N.; Grace, J.R.; Bi, H.T. Layer inversion phenomenon in binary-solid liquid-fluidized beds: Prediction of the inversion velocity. Chem. Eng. Sci. 2006, 61, 6667-6690. [CrossRef]

16. Epstein, N.; Pruden, B.B. Liquid fluidisation of binary particle mixtures-III Stratification by size and related topics. Chem. Eng. Sci. 1999, 54, 401-415. [CrossRef]

17. Gibilaro, L.G.; Di Felice, R.; Waldram, S.P.; Foscolo, P.U. A predictive model for the equilibrium composition and inversion of binary-solid liquid fluidized beds. Chem. Eng. Sci. 1986, 41, 379-387. [CrossRef]

18. Asif, M. Predicting binary-solid fluidized bed behavior using averaging approaches. Powder Technol. 2002, 127, 226-238. [CrossRef]

19. Funamizu, N.; Takakuwa, T. An improved Richardson-Zaki formula for computing mixed layer composition in binary solid-liquid fluidized beds. Chem. Eng. Sci. 1995, 50, 3025-3032. [CrossRef]

20. Di Maio, F.P.; Di Renzo, A. Direct modeling of voidage at layer inversion in binary liquid-fluidized bed. Chem. Eng. J. 2016, 284, 668-678. [CrossRef]

21. Van der Hoef, M.A.; Beetstra, R.; Kuipers, J.A.M. Lattice-Boltzmann simulations of low-Reynolds-number flow past mono- and bidisperse arrays of spheres: Results for the permeability and drag force. J. Fluid Mech. 2005, 528, 233-254. [CrossRef]

22. Yin, X.; Sundaresan, S. Fluid-particle drag in low-Reynolds-number polydisperse gas-solid suspensions. AIChE J. 2009, 55, 1352-1368. [CrossRef]

23. Cello, F.; Di Renzo, A.; Di Maio, F.P. A semi-empirical model for the drag force and fluid-particle interaction in polydisperse suspensions. Chem. Eng. Sci. 2010, 65, 3128-3139. [CrossRef]

24. Rong, L.W.; Dong, K.J.; Yu, A.B. Lattice-Boltzmann simulation of fluid flow through packed beds of spheres: Effect of particle size distribution. Chem. Eng. Sci. 2014, 116, 508-523. [CrossRef]

25. Escudié, R.; Epstein, N. Voidage at the Layer Inversion Point in Binary-Solid Liquid-Fluidized Beds. Ind. Eng. Chem. Res. 2009, 48, 182-184. [CrossRef]

(C) 2020 by the authors. Licensee MDPI, Basel, Switzerland. This article is an open access article distributed under the terms and conditions of the Creative Commons Attribution (CC BY) license (http://creativecommons.org/licenses/by/4.0/). 